\title{
FOLFOX plus cetuximab in first-line therapy of advanced colorectal cancer
}

\author{
Roland Ehrenberg, Niels Halama \\ National Center for Tumor Diseases, Department of Medical Oncology, University Hospital Heidelberg, Heidelberg, Germany \\ Correspondence to: Niels Halama. National Center for Tumor Diseases, Department of Medical Oncology, University Hospital Heidelberg, Im \\ Neuenheimer Feld 460, Heidelberg, Germany. Email: Niels.Halama@nct-heidelberg.de. \\ Comment on: Qin S, Li J, Wang L, et al. Efficacy and Tolerability of First-Line Cetuximab Plus Leucovorin, Fluorouracil, and Oxaliplatin (FOLFOX-4) \\ Versus FOLFOX-4 in Patients With RAS Wild-Type Metastatic Colorectal Cancer: The Open-Label, Randomized, Phase III TAILOR Trial. J Clin \\ Oncol 2018. [Epub ahead of print].
}

Submitted Oct 29, 2018. Accepted for publication Nov 09, 2018.

doi: $10.21037 / \mathrm{atm} .2018 .11 .22$

View this article at: http://dx.doi.org/10.21037/atm.2018.11.22

Oxaliplatinum is derivate of platinum, which has been used for colorectal cancer since 1997 in France for first-line therapy of metastatic colorectal cancer. Following the data from the MOSAIC trial (1), oxaliplatinum-based adjuvant therapy was established. Development of the oxaliplatinumbased protocols lead to the development of FOLFOX4 (2) and FOLFOX6 (3) and subsequent FOLFOX7 protocols (4). Oral formulations of 5-FU as capecitabine (Xeloda) were tested and showed non-inferiority to infusional regimens $(5,6)$ also in second line settings $(7)$ and in combination with oxaliplatinum. The TRIBE trial compared FOLFOXIRI plus bevacizumab $v s$. FOLFIRI plus bevacizumab (8) where FOLFOXIRI plus bevacizumab yielded a median overall survival (OS) 29.8 months with a benefit of bevacizumab across all analyzed mutational profiles. In contrast, the PRIME trial (9) used prospective analysis of the KRAS status and half of the patients received additionally panitumumab (combination arm), whereas all received FOLFOX4. In this trial of previously untreated colorectal cancer patients, 593 patients were in arm 1 (combination) and 590 in arm 2 (with only FOLFOX4 treatment). In $93 \%$ was KRAS status analyzed. Final analyses showed a median progression-free survival (PFS) with panitumumab of 10.0 months versus 8.6 months without. No difference in OS was noted, but there was a clear-but not statistically significant—difference of 23.9 versus 19.7 months observed. The response rates were significant with $57 \%$ versus $48 \%$. Patients with KRAS mutation had reduced PFS and OS in combination with panitumumab, whereas KRAS wildtype showed better survival (25.8 versus 20.7 months). In this cohort, further analyses showed ECOG 0/1 patients to benefit most from combination therapy, in the KRAS wildtype cohort. The PEAK trial compared FOLFOX plus panitumumab versus FOLFOX plus bevacizumab in first-line therapy and revealed the combination with panitumumab to be better for KRAS wildtype patients (13.0 versus 9.5 months median PFS, $\mathrm{P}=0.029$ ) (10). Analysis of the data from the OPUS trial (11) showed for the FOLFOX plus cetuximab combination detrimental outcomes for patients with tumors with KRAS mutations [i.e., worse PFS and objective response rate (ORR)]. In the wildtype population the median PFS was 12.0 versus 5.8 months, only with a trend for significance $(\mathrm{P}=0.0615)$. The median OS was 19.8 versus 17.8 months $(\mathrm{P}=0.8)$. The described trials therefore have not fully clarified the role of FOLFOX plus cetuximab in first line therapy of colorectal cancer patients with wildtype $R A S$ status. In this situation, the data from the TAILOR study try to close the gap. The TAILOR trial is an open-label, randomized, phase III trial in patients from China comparing FOLFOX-4 with or without cetuximab in RAS wildtype metastatic colorectal cancer. For mutational status, KRAS/NRAS, exons 2 to 4 were investigated, allowing to assess the majority of presenting RAS mutations in patients. The primary endpoint of the TAILOR trial was progressionfree survival time; secondary end points included OS time, overall response rate as well as safety and tolerability. In the modified intent-to-treat (mITT) population of a set of 393 patients with RAS wildtype, addition of cetuximab to FOLFOX-4 significantly improved the primary end 
point of progression-free survival time compared with FOLFOX-4 alone. Hazard ratio was 0.69 with a $95 \%$ confidence interval (CI) of 0.54 to $0.89, \mathrm{P}=0.004$, revealing a median PFS of 9.2 versus 7.4 months. The secondary end point of OS time was assessed after 300 events and a hazard ratio of 0.76 was observed (95\% CI: 0.61-0.96, $\mathrm{P}=0.02$ ), resulting in a median $\mathrm{OS}$ of 20.7 versus 17.8 months. The overall response rate was also significantly different between the two arms (with an odds ratio of 2.41 and a $95 \%$ CI: $1.61-3.61, \mathrm{P}=0.001): 61.1 \%$ versus $39.5 \%$ respectively. The treatment was well tolerated and no new or unexpected safety issues were observed. The authors report that their inclusion criteria were changed from patients with KRAS exon 2 analyses to patients with extended RAS analysis (KRAS/NRAS exons 2 to 4 ) being performed on their tumors. The final analysis was based on this mITT population. This might explain the observed differences in OS in this Chinese patient cohort. A total of 20.7 months OS seems to be quite short in comparison to published data [e.g., FIRE-3 data, Heinemann et al. (12), Lancet Oncology] with over 30 months survival. The authors explain this obvious difference with the limited access to oncological substances and oncological care for the investigated patient cohort. The possible inclusion of patient with a RAS/RAF mutational profile beyond the analyzed exon 2 mutations in the initial phase of the trial could further skew the data in an unfavorable manner. Without a retrospective analysis of the included patients, this question cannot be answered. Another aspect that might also contribute to a certain difference between the combination arm and the chemotherapy arm is the balance of metastatic sites in the patients from the two treatment arms. The combination arm has $20.7 \%$ with three or more sites of metastases while the chemotherapy only arm has $28.5 \%$ of patients with three or more sites involved. Of course, a possibility would be the presentation of volumetric tumor burden for the patients in the trial. Another interesting aspect in comparison to the FIRE-3 data is the sidedness of the primary localization of the tumor. In the TAILOR trial, close $80 \%$ of the tumors in the combination arm were located on the left side of the colon. It would be interesting-also in the light of the data from the FIRE3 trial-to see the sidedness in relation to the treatment outcome in the TAILOR trial population. Another intriguing aspect of the findings observed in the TAILOR trial is the finding, that FOLFOX4 therapy appears to be more beneficial for the BRAF mutant subgroup compared to the combination therapy. This is feeding an ongoing debate, whether or not the addition of EGFR-targeted agents is helpful for BRAF mutated colorectal cancer. Two meta-analyses came to differing conclusions [Rowland et al. (13) versus Pietrantonio et al. (14)]: one concluded that there is insufficient evidence to justify withholding antiEGFR therapy, the other discouraged the use of anti-EGFR in these patients. More analyses in this direction are clearly needed and currently promising data from the VOLFI trial on the combination of FOLFOXIRI plus panitumumab in BRAF mutated tumors could be reported (15). In terms of toxicities, there were no unexpected findings. Of note, leukopenia and neutropenia in the TAILOR trial were more frequent than reported in previous studies. An explanation for this observation is missing. Apart from this, the TAILOR trial now provides more evidence for the debate around cetuximab plus FOLFOX combinations, corroborating the data from the OPUS trial and setting the ground for acceptable routine use of this combination treatment.

Beyond this, Oxaliplatinum has a long history and is well established in the clinic. Recent data from immunotherapy trials (e.g., NICHE trial, Abstract LBA37_PR 'Neoadjuvant ipilimumab plus nivolumab in early stage colon cancer' by Myriam Chalabi, Annals of Oncology, Volume 29 Supplement 8 October 2018) suggest, that it might be more beneficial to apply immunotherapy in earlier stage of the disease. In addition, Oxaliplatinum activity on tumor cells shows immune-stimulating features, most notably immunogenic cell death $(16,17)$ and is also associated with the presence of infiltrating immune cells in the local microenvironment (18). As we see more and more (earlier) immunotherapeutic approaches for microsatellite-stable and microsatelliteinstable colorectal cancers in trials, combinatorial approaches are not in the clinic yet. But the presented data suggests the possibility for synergisms and there might be a role for oxaliplatinum-based combination immunotherapies in the future for colorectal cancer.

\section{Acknowledgements}

None.

\section{Footnote}

Conflicts of Interest: The authors have no conflicts of interest to declare. 


\section{References}

1. Andre T, Boni C, Mounedji-Boudiaf L, et al. Oxaliplatin, fluorouracil, and leucovorin as adjuvant treatment for colon cancer. N Engl J Med 2004;350:2343-51.

2. Andre T, Bensmaine MA, Louvet C, et al. Multicenter phase II study of bimonthly high-dose leucovorin, fluorouracil infusion, and oxaliplatin for metastatic colorectal cancer resistant to the same leucovorin and fluorouracil regimen. J Clin Oncol 1999;17:3560-8.

3. Maindrault-Goebel F, Louvet C, Andre T, et al. Oxaliplatin added to the simplified bimonthly leucovorin and 5-fluorouracil regimen as second-line therapy for metastatic colorectal cancer (FOLFOX6). GERCOR. Eur J Cancer 1999;35:1338-42.

4. Maindrault-Goebel F, de Gramont A, Louvet C, et al. High-dose intensity oxaliplatin added to the simplified bimonthly leucovorin and 5-fluorouracil regimen as second-line therapy for metastatic colorectal cancer (FOLFOX 7). Eur J Cancer 2001;37:1000-5.

5. Saltz LB, Clarke S, Diaz-Rubio E, et al. Bevacizumab in combination with oxaliplatin-based chemotherapy as firstline therapy in metastatic colorectal cancer: a randomized phase III study. J Clin Oncol 2008;26:2013-9.

6. Cassidy J, Clarke S, Diaz-Rubio E, et al. Randomized phase III study of capecitabine plus oxaliplatin compared with fluorouracil/folinic acid plus oxaliplatin as first-line therapy for metastatic colorectal cancer. J Clin Oncol 2008;26:2006-12.

7. Rothenberg ML, Cox JV, Butts C, et al. Capecitabine plus oxaliplatin (XELOX) versus 5-fluorouracil/folinic acid plus oxaliplatin (FOLFOX-4) as second-line therapy in metastatic colorectal cancer: a randomized phase III noninferiority study. Ann Oncol 2008;19:1720-6.

8. Loupakis F, Cremolini C, Masi G, et al. Initial therapy with FOLFOXIRI and bevacizumab for metastatic colorectal cancer. N Engl J Med 2014;371:1609-18.

9. Douillard JY, Siena S, Cassidy J, et al. Randomized, phase III trial of panitumumab with infusional fluorouracil, leucovorin, and oxaliplatin (FOLFOX4) versus FOLFOX4 alone as first-line treatment in patients with previously untreated metastatic colorectal cancer: the PRIME study. J

Cite this article as: Ehrenberg R, Halama N. FOLFOX plus cetuximab in first-line therapy of advanced colorectal cancer. Ann Transl Med 2018;6(Suppl 2):S96. doi: 10.21037/ atm.2018.11.22
Clin Oncol 2010;28:4697-705.

10. Schwartzberg LS, Rivera F, Karthaus M, et al. PEAK: a randomized, multicenter phase II study of panitumumab plus modified fluorouracil, leucovorin, and oxaliplatin (mFOLFOX6) or bevacizumab plus mFOLFOX6 in patients with previously untreated, unresectable, wild-type KRAS exon 2 metastatic colorectal cancer. J Clin Oncol 2014;32:2240-7.

11. Bokemeyer C, Bondarenko I, Hartmann JT, et al. Efficacy according to biomarker status of cetuximab plus FOLFOX-4 as first-line treatment for metastatic colorectal cancer: the OPUS study. Ann Oncol 2011;22:1535-46.

12. Heinemann V, von Weikersthal LF, Decker T, et al. FOLFIRI plus cetuximab versus FOLFIRI plus bevacizumab as first-line treatment for patients with metastatic colorectal cancer (FIRE-3): a randomised, open-label, phase 3 trial. Lancet Oncol 2014;15:1065-75.

13. Rowland A, Dias MM, Wiese MD, et al. Meta-analysis of BRAF mutation as a predictive biomarker of benefit from anti-EGFR monoclonal antibody therapy for RAS wild-type metastatic colorectal cancer. Br J Cancer 2015;112:1888-94.

14. Pietrantonio F, Petrelli F, Coinu A, et al. Predictive role of BRAF mutations in patients with advanced colorectal cancer receiving cetuximab and panitumumab: a metaanalysis. Eur J Cancer 2015;51:587-94.

15. Geissler M, Riera-Knorrenschild J, Tannapfel A, et al. mFOLFOXIRI + panitumumab versus FOLFOXIRI as first-line treatment in patients with RAS wild- type metastatic colorectal cancer $\mathrm{m}(\mathrm{CRC})$ : A randomized phase II VOLFI trial of the AIO (AIO- KRK0109). J Clin Oncol 2018;36:3509.

16. Tesniere A, Schlemmer F, Boige V, et al. Immunogenic death of colon cancer cells treated with oxaliplatin. Oncogene 2010;29:482-91.

17. Zitvogel L, Kepp O, Kroemer G. Immune parameters affecting the efficacy of chemotherapeutic regimens. Nat Rev Clin Oncol 2011;8:151-60.

18. Halama N, Michel S, Kloor M, et al. Localization and Density of Immune Cells in the Invasive Margin of Human Colorectal Cancer Liver Metastases Are Prognostic for Response to Chemotherapy. Cancer Res 2011;71:5670-7. 